\title{
Occupational exposure to zeranol, an animal growth promoter
}

\author{
T C AW,' A B SMITH,' R L STEPHENSON,' C J GLUECK ${ }^{2}$
}

From the National Institute of Occupational Safety and Health, ${ }^{\prime}$ Cincinnati, Ohio 45226, and the Cholesterol Center, ${ }^{2}$ Jewish Hospital, Cincinnati, Ohio 45229, USA

\begin{abstract}
Zeranol (3,4,5,6,7,8,9,10,11,12-Decahydro-7,14,16-trihydroxy-3-methyl-1H-2-benzoxacyclotetradecin-1-one) is a synthetic oestrogenic agent used as an animal growth promoter. The effects of occupational exposure to zeranol in 11 exposed workers from a pelletising plant and 14 nonexposed subjects were assessed. A questionnaire showed that more breast symptoms were reported by male and female plant workers compared with non-exposed subjects, although the difference was not statistically significant. Clinical assessment showed no cases of gynaecomastia in all the male participants. Blood samples analysed by high performance liquid chromatography for zeranol, its precursor zearalenone, and its main metabolites did not show any of these compounds above the laboratory limit of detection. Serum levels of follicle stimulating hormone (FSH), luteinising hormone $(\mathrm{LH})$, prolactin, and oestradiol showed no striking differences between the exposed and the non-exposed subjects. Total and low density lipoprotein cholesterol (LDL cholesterol) levels did not significantly differ between the two groups but mean high density lipoprotein cholesterol (HDL cholesterol) levels were higher in the exposed group; this could be due to relatively high HDL cholesterol in two women exposed to zeranol or relatively low HDL cholesterol in three non-exposed men.
\end{abstract}

Zeranol [CAS No 55331-29-8] is a synthetic, nonsteroidal, anabolic agent that also has oestrogenic activity. ${ }^{1-6}$ Its chemical structure $(3,4,5,6,7,8,9,10,11$, 12-Decahydro-7, 14, 16-trihydroxy-3-methyl-1 H-2-benzoxacyclotetradecin-1-one) is shown in the figure. It is produced commercially for use in animal husbandry to accelerate weight gain in beef cattle and feedlot lambs before slaughter. In animal models zeranol can apparently induce many of the same biological and biochemical responses as are evoked by oestradiol. ${ }^{5}$ Its oestrogenic properties are used to treat menopausal symptoms in women ${ }^{78}$ and it has been sold as a pharmaceutical agent for this purpose in some European countries, although not in the United Kingdom or the United States.

Zeranol is the most widely used animal growth promoter in the United States. The use of animal growth promoters has given rise to various public health concerns, ${ }^{9-12}$ particularly since synthetic nonsteroidal oestrogens such as diethylstilboestrol, which was used as an animal growth promoter, were shown to be carcinogenic. ${ }^{13}$ Zearalenone, the precursor in the manufacture of zeranol, is an oestrogenic mycotoxin with teratogenic, carcinogenic, and mutagenic properties. ${ }^{14-17}$ It has been reported to be mutagenic for strains of Bacillus subtilis ${ }^{17}$ but not Salmonella typhimurium..$^{1519}$ A review on zeranol itself did not indicate similar properties. ${ }^{2}$ Human exposure to zeranol could occur through ingestion of animal products which contain pharmacologically active residual zeranol or its metabolites, or by the therapeutic use of zeranol to alleviate postmenopausal

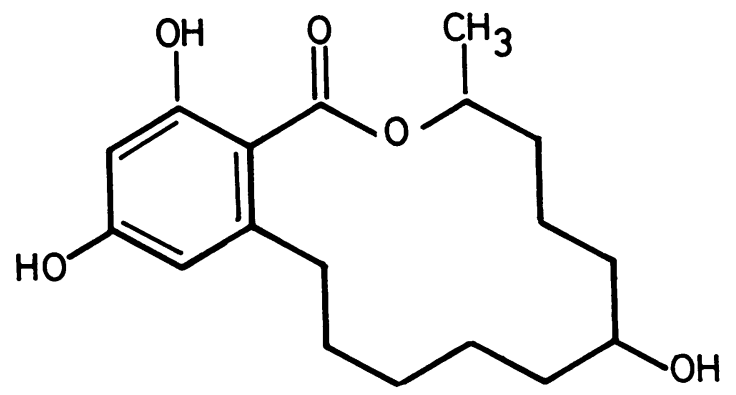

Zeranol. $^{1}$ 
symptoms, or through industrial or environmental exposure.

In 1982 we were asked to assess the effects of gross industrial exposure to zeranol in workers from a small manufacturing plant which formulates, pelletises, and packages an animal growth promoter containing zeranol as its active ingredient. ${ }^{20}$ The request resulted from the reporting of breast symptoms among plant workers and the observation that two former workers (one man, one woman) each had a boy aged under 5 years who developed gynaecomastia and had an unusual growth spurt while their parents were still employed at the plant. One child had an extensive clinical evaluation, which additionally showed an advanced bone age. Exposure to zeranol occurred because the parents wore their workclothes home. Clinical features in both children decreased after the parents left their jobs at the plant. Similar effects have previously been reported in workers handling oestrogens at a plant manufacturing oral contraceptives. ${ }^{21}$

The present study was conducted after new construction nearly doubled the floor space of the existing plant and after some work practices had been improved. Specifically, disposable air purifying dust respirators were made available, workers were provided with workclothes and the laundering of such, and an attempt was made to provide local exhaust ventilation for some of the work processes in the packaging area.

\section{Materials and methods}

\section{SUBJECTS}

Eleven workers (6 women, 5 men) were directly exposed to the zeranol powder in the manufacturing plant. For comparison, 14 clerical and professional staff members of a government office (6 women, 8 men) in the same geographical area were evaluated. This comparison group had had no contact or exposure to zeranol.

The exposed workers included five production and packaging personnel who had regular direct skin contact with zeranol either during mixing and sifting of zeranol powder or during manual packaging of the finished pellets into plastic containers. The other six exposed workers were administrative, laboratory, and cleaning staff with intermittent contact with zeranol powder or finished pellets. Exposure to zeranol was therefore primarily by skin contact and inhalation; there was no exposure to the precursor zearalenone.

\section{ENVIRONMENTAL HYGIENE MEASUREMENTS}

To measure exposure to zeranol, personal air samples were taken for three days in the work area and other parts of the plant. The samples were collected on to Millipore Fluoropore polytetrafluoroethylene type
FHLP filters $(0.5$ microns, $37 \mathrm{~mm})$ with backup pads Surface wipe samples of the lunch room, ventilation system, and work surfaces were also collected. Cotton: gauze pads backed by aluminium foil were attached by plastic straps to the palms of the workers to assess the extent of skin contamination. All the wipe samples and hand gauze pad samples were collected on $25 \mathrm{~cm} \%$. cotton pads wetted with isopropanol. Analysis of al居 environmental hygiene samples was by use of a high performance liquid chromatograph (HPLC) equippe\& with an ultraviolet (UV) detector. Six to nine aip samples were taken in the production, packaging, and laboratory area. Five to nine dermal wipe samplefs were taken in the same areas.

\section{MEDICAL INTER VIEWS AND CLINICAL} ASSESSMENTS

The protocol used was similar to one suggested foi investigating workers in the pharmaceutical industry exposed to synthetic oestrogens. ${ }^{22}$ All exposed an $\Phi$ non-exposed workers were interviewed using a ques=tionnaire focusing on occupational and medical his 3 tory. The occurrence of breast symptoms and othe symptoms relevant to oestrogenic effects in the work $\vec{\overrightarrow{ }}$ ers was determined. The children were not clinicallog examined, but information on whether they have had breast problems requiring medical advice was sough from the questionnaire.

To determine if gynaecomastia was present in adu male participants, a clinical assessment was performe by a physician with no prior knowledge of the identite of those examined. This "blind" assessment wa limited to examination of the anterior chest wall. The case definition used for gynaecomastia was "a palp? able, discrete button of firm subareolar tissue measu? ing at least $2 \mathrm{~cm}$ in diameter." ${ }^{23}$ Female participant8 were not examined.

Non-fasting serum samples were obtained from eight of the exposed and 12 of the unexposed subjects. Determination of zeranol, its main metabolites, and its precursor was made using a high performance liqui $\$$ chromatography method. The limit of detection fop zeranol was $12 \cdot 2 \mu \mathrm{g} / \mathrm{l}$, zearalenone $27 \cdot 1 \mu \mathrm{g} / 1$, zearala none $18.8 \mu \mathrm{g} / \mathrm{l}$, and taleranol $11.5 \mu \mathrm{g} / \mathrm{l}$.

Serum follicle stimulating hormone (FSH), luteinis ing hormone $(\mathrm{LH})$, prolactin, and oestradiol were measured by radioimmunoassay. To determinte whether female subjects were at the folliculaț ovulatory, or luteal phase of the menstrual cycle at the time of blood collection, they were re-interviewey eight weeks after blood collection and asked for the dates of their last menstrual cycles. The serum FSE and LH were compared with the laboratory's normat range for the appropriate phase of the cycle. Seruro oestradiol for male subjects was determined b radioimmunoassay of random blood sample. 
Lipid and lipoprotein cholesterol measurements were made using lipids research clinics (LRC) methodology $^{24}$ in the LRC standardised Cincinnati core laboratory.

\section{Results}

WORK EXPOSURE TO ZERANOL

One year before the present study, the company conducted air monitoring for zeranol in the production department and found that exposures ranged from 683 to $4000 \mu \mathrm{g} / \mathrm{m}^{3}$. In the present study the highest airborne zeranol concentrations were also in the production area (table 1) with a mean of $584 \mu \mathrm{g} / \mathrm{m}^{3}$ and a range of 86 to $1554 \mu \mathrm{g} / \mathrm{m}^{3}$. Cotton gauze pad samples indicated the highest skin contamination by zeranol to be in the production department. Surface wipe samples showed zeranol contamination in the lunchroom (median of $0.4 \mathrm{mg}$ zeranol/sample), the laboratory $(0.2 \mathrm{mg} / \mathrm{sample})$, and inside a ceiling air supply duct of the plant ventilation system $(5.1 \mathrm{mg} /$ sample). Sixteen milligrams of zeranol powder was detected on one half of a work garment that had been worn by a packager for two consecutive days. Doubling that amount to account for the other half of the garment provides an estimate of $32 \mathrm{mg}$ of zeranol on the work garment to which the packager was exposed. This is more than the amount present in one pellet of the finished product $(12 \mathrm{mg})$ and slightly less than the dose that would be administered subcutaneously to a steer $(36 \mathrm{mg})$.

\section{QUESTIONNAIRE AND CLINICAL ASSESSMENTS}

The unexposed group were younger (mean age 27) than the exposed (mean age 33). Alcohol consumption of more than five drinks a week was higher in the comparison group $(29 \%)$ than the exposed group $(9 \%)$. No past history of endocrine disorders was present in either group. Racial composition, smoking experience, and Quetelet index were comparable.

Six of the 11 exposed workers, compared with three of 14 non-exposed, reported at least one of the following: sharp pain, tingling, burning, aching, or irritation of the breasts. The numbers are small and the difference was not statistically significant. Two each of the women exposed to zeranol and women not exposed reported spotting between menstrual periods since starting their current job. One woman exposed to zeranol reported having irregular periods since starting her present job. None of the participants (exposed or non-exposed) reported impotence, loss of interest in sex, or any perceived infertility. Six current workers exposed to zeranol had a total of 15 children living at home ( 11 boys, four girls). Three boys had had breast enlargement and discomfort and the mothers had sought medical advice. (These three boys do not include the two boys of the former workers whose symptoms led to the present study.) One boy's symptoms appeared to be due to pubertal changes. The symptoms in the two other boys abated when modifications were made at the plant to control exposures. None of the children had any specific laboratory investigations undertaken. Four of the comparison participants had a total of six children living at home; none had any known breast or other health problems.

Clinical assessment of the male participants showed no evidence of subareolar nodular masses in the breasts in either group. Laboratory analyses for zeranol and its analogues showed no zeranol, zearalenone, zearalanone, or taleranol in the serum samples above the laboratory limit of detection.

Pregnant women and those taking oral contraceptives were excluded from further analysis. LH levels were within the normal range for all participants. FSH levels were within normal limits for all participants exposed to zeranol and marginally outside laboratory normal limits in three non-exposed participants. Two of these FSH levels were lower, and one was higher than normal. Prolactin levels were within normal limits for all participants. All men had serum oestradiol levels within the laboratory's normal range, except for one worker exposed to zeranol who had a marginally raised level. The mean $( \pm$ standard deviation) oestradiol concentrations for the men exposed to zeranol was $39 \pm 20 \mathrm{ng} / \mathrm{l}$, compared with $41 \pm 13 \mathrm{ng} / \mathrm{l}$ for the men not exposed.

Covariance adjusted (for age, sex, and alcohol consumption) total cholesterol and LDL cholesterol levels did not significantly differ in the two groups (table 2). Specimens were drawn non-fasting, so triglyceride levels were not specifically examined.

Table 1 Zeranol detected by environmental hygiene measurements

\begin{tabular}{|c|c|c|c|c|c|c|c|c|}
\hline \multirow[b]{2}{*}{ Work area } & \multicolumn{4}{|c|}{ Air samples $\left(\mu g / m^{3}\right)$} & \multicolumn{4}{|c|}{ Gauze pad samples (mg/sample*) } \\
\hline & No & Mean & Median & Range & No & Mean & Median & Range \\
\hline $\begin{array}{l}\text { Production } \\
\text { Packaging } \\
\text { Laboratory }\end{array}$ & $\begin{array}{l}6 \\
8 \\
9\end{array}$ & $\begin{array}{r}584 \\
67 \\
9\end{array}$ & $\begin{array}{r}547 \\
46 \\
7\end{array}$ & $\begin{array}{rr}86-1554 \\
30- & 145 \\
4 & 21\end{array}$ & $\begin{array}{l}5 \\
9 \\
9\end{array}$ & $\begin{array}{c}133 \\
1.4 \\
0.5\end{array}$ & $\begin{array}{r}90.0 \\
1.3 \\
0.3\end{array}$ & $\begin{array}{l}1.1-410 \\
0.04-2.3 \\
0.004-1.7\end{array}$ \\
\hline
\end{tabular}

*Each sample being collected on a $25 \mathrm{~cm}^{2}$ cotton gauze pad wetted with isopropanol. 
Table 2 Covariance adjusted means for lipids and lipoprotein cholesterols ( $\mathrm{mg} / \mathrm{l})$ in zeranol exposed and non-exposed workers

\begin{tabular}{|c|c|c|c|c|c|}
\hline Group & No & Age & Total cholesterol & $H D L C$ & $L D L C$ \\
\hline $\begin{array}{l}\text { Exposed } \\
\text { Non-exposed } \\
\text { p Value* }\end{array}$ & $\begin{array}{l}8 \\
9\end{array}$ & $\begin{array}{l}35 \pm 3 \\
28 \pm 2\end{array}$ & $\begin{array}{c}2020(5.22 \mathrm{mmol} / \mathrm{l}) \\
1900(4.91 \mathrm{mmol} / \mathrm{l}) \\
0.73\end{array}$ & $\begin{array}{l}630(1.63 \mathrm{mmol} / \mathrm{l}) \\
340(0.88 \mathrm{mmol} / \mathrm{l}) \\
0.03\end{array}$ & $\begin{array}{c}1150(2.97 \mathrm{mmol} / \mathrm{l}) \overline{0} \\
1140(2.95 \mathrm{mmol} / \mathrm{l}) \\
0.98\end{array}$ \\
\hline
\end{tabular}

*Significance of effect of exposure, covariance adjusted for age, sex, and alcohol consumption.

Covariance adjusted mean HDL cholesterol levels were higher in exposed than non-exposed subjects but on examination of the individual data, it was noted that the significance of the regression could reasonably be explained either by relatively high HDL cholesterol levels in two women exposed to zeranol or by relatively low HDL cholesterol levels among three non-exposed men. All five determinations were in the respective tails of the HDL cholesterol age and sex specific distributions cited by the lipid research clinics for the United States and Canada. ${ }^{25}$ The two alternatives cannot be resolved through further analyses of these data alone.

\section{Discussion}

Only 11 workers were exposed to zeranol and when women who were pregnant or taking oral contraceptives were deleted from analysis, the number of exposed workers fell to eight. Thus the statistical power to detect anything other than substantial differences between exposed and non-exposed workers is small. None the less, these workers represented the total current population of workers in the United States pelletising and packaging an animal growth promoter containing zeranol.

The exposed workers had gross skin contamination with the zeranol powder, and there was exposure by inhalation and probably involuntary ingestion as well. This may occur as a result of the contamination of the ventilation system, lunch room, and workclothes, especially when gloves and respirators were not used. The precursor of zeranol may be absorbed through intact skin of the rat and oestrogenic effects have been produced by this route of exposure. ${ }^{26}$ The history of gynaecomastia in two children of former workers provided strong clinical evidence of hyperoestrogenism as a consequence of workplace exposures two years before our study which, after plant redesign and work practice modification had taken place, was generally negative or equivocal in documenting oestrogenic effects among the exposed workers.

An excess of breast symptoms was reported among male and female workers exposed to zeranol but the numbers were small and differences insignificant. Harrington has suggested that intermenstrual bleeding is the most significant feature in women oc- cupationally exposed to exogenous oestrogens $\mathrm{s}^{22}$; w5 found no difference between exposed and non-exposed women in this regard. Nor was there impotence, loss oft. libido, or gynaecomastia among the men, exposed of non-exposed.

Serum LH and FSH levels in the exposed female workers were all within normal limits for the estimate phase of the menstrual cycle. Since LH and FSH are्ट thought to be secreted in a spiked manner several times a day, however, single measurements of these gonao dotrophins may not yield representative values. ${ }^{27}$ In addition, the effects of oestrogens on $\mathrm{LH}$ and FSH levels at low levels of exposure may be subtle and map be detectable only by repeat sampling over the course. of the menstrual cycle or by tests of gonadotrophiro reserve in response to challenge by the appropriate hypophyseal releasing hormones. ${ }^{28}$ Such procedure are impractical in an industrial survey.

There were no differences in serum prolactin, oes tradiol, total cholesterol, or in LDL cholesterol level: between the groups. Although HDL cholesterol level\$ differed between the exposed and non-exposed, we cannot rule out the possibility that HDL cholesterof levels were unusually depressed in the control group ot that the two women with raised HDL cholesterol mat have been unusual.

We speculate that our data may be useful ip estimating a provisional "no clinical effect" level for occupational exposure to zeranol. The mean aiß concentration of zeranol in the production area was. $584 \mu \mathrm{g} / \mathrm{m}^{3}$. Multiplying by an estimated 10 cubie metres of air inhaled over eight hours by workers with moderate exertion gives a daily airborne exposure of $5.8-7 \mathrm{mg}$ of zeranol a day for production workers. $\frac{29}{O}$ The equivalent calculation for packaging workers is. between 0.7 and $0.8 \mathrm{mg}$. We estimated $32 \mathrm{mg}$ to be on contaminated work garment worn by a packaging worker. The dermal wipe samples revealed $133 \mathrm{mg}$ foR a production worker. How does this compare with the estimated minimally effective biological dose of zeranol?

Zeranol was sold in Europe under a different trad为 name for treatment of postmenopausal symptoms in women. In one study $50 \mathrm{mg}$ was determined to be minimally effective dose for producing changes ip maturation of vaginal corneal epithelium, wherea\& 
$100 \mathrm{mg}$ a day was necessary to show an effect on the hypophyseal gonadotrophins. ${ }^{30}$ Data on non-human primates extrapolated to a $70 \mathrm{~kg}$ person indicate that the minimally effective oral dose of the precursor zearalenone needed to produce changes in $\mathrm{LH}$ secretion is $28 \mathrm{mg}{ }^{31}$ For zeranol, the no oestrogenic effect level extrapolated to a $70 \mathrm{~kg}$ person has been estimated by scientists with the United States manufacturer as $15 \mathrm{mg} .^{2}$

Our estimated maximum dose of $5 \cdot 8-7 \mathrm{mg}$ a day by the airborne route is less than the lowest effective dose estimated by others. ${ }^{2}$ The amounts on workclothes and skin contamination are, however, equal to or greater than the estimated minimally effective oral dose. For systemic zeranol absorption at the time of our study to have been above the lowest effective level estimated from published reports, some absorption of zeranol through skin or workclothes would have to have occurred. Despite documentation of skin and clothing contamination, however, zeranol and its analogues were not detected in blood specimens in all workers. This suggests that unlike zearalenone absorption in rats, ${ }^{26}$ absorption of zeranol through the intact skin may not contribute significantly to systemic absorption in man.

There was no difference in prolactin concentrations between exposed and non-exposed subjects. The minimum effective oral dose of ethinyl oestradiol required to stimulate prolactin secretion in regularly menstruating women is about $100 \mathrm{mg}$ a day. ${ }^{31}$ If zeranol is 100 times less potent than ethinyl oestradiol by weight, ${ }^{32}$ then about $10 \mathrm{mg}$ of zeranol by mouth would be required daily to stimulate prolactin production. This level is greater than our upper estimate of 7 $\mathrm{mg}$ a day of zeranol exposure through the airborne route. Comparisons between ingested and inhaled doses of the same substance, however, may be difficult, especially when there is little information on the difference in biological activity when exposure and absorption are by different routes.

Occupational exposure to synthetic oestrogens may have other health related ramifications, particularly in regard to risks of cancer and to abnormal growth and development in childhood. Long term exogenous exposure to oestrogens is thought to increase the risk of cancer of the breast, ${ }^{33}$ and possibly of the cervix and uterus. ${ }^{34}$ Contamination of the home environment of exposed workers may cause secondary exposure to their children. Exogenous oestrogens in both boys and girls before, or at, early pubescence may lead to an effect on bone growth. ${ }^{35}$ At this plant producing the zeranol based animal growth promoter, recommendations were made to reduce exposure to the zeranol dust and to minimise the possibility of the compound being brought home on workclothes. Any likely long term effects of zeranol in the exposed workers may be determined by continued medical surveillance of the exposed workers augmented by environmental monitoring to confirm reduced exposures.

This study was carried out following a protocol approved by the Human Subjects Review Board of the National Institute for Occupational Safety and Health.

Requests for reprints to: Dr Tar-Ching Aw, Institute of Occupational Health, University of Birmingham, University Road West, PO Box 363, Birmingham, B15 2TT, UK.

\section{References}

1 Windholz M, Budavari S, Blumetti RF, Otterbein ES, eds. Merck index. 10th ed. Rahway, NJ: Merck \& Co, 1983.

2 Baldwin RS, William RD, Terry MK. Zeranol: a review of the metabolism, toxicology, and analytical methods for detection of tissue residues. Regul Toxicol Pharmacol 1983;3:9-25.

3 O'Lamhna MP, Roche JF. Effect of long- or short-acting anabolic agents, given singly or repeated, on growth rate and carcass weight of steers. Vet Rec 1984;114:182-4.

4 O'Lamhna MP, Roche JF. Recent studies with anabolic agents in steers and bulls. Current Topics in Veterinary Animal Science, ISS Manipulation Growth Farm Animals 1984;26:85-99.

5 Kitts WD, Newsome FE, Funeckles VC. The estrogenic and antiestrogenic effects of coumestrol and zearalanol on the immature rat uterus. Canadian Journal of Animal Sciences 1984;63:823-34.

6 O'Lamhna MP, Roche JF. Effect of repeated implantation with anabolic agents on growth rate, carcass weight, testicular size and behavior of bulls. Vet Rec 1983;113:531-4.

7 Seppala M, Vara P. Zeranol (P1496), a nonsteroid analog, in long term treatment of climacteric syndrome. Ars Medici (Leistal) 1976;66:467-70.

8 Utian WH. Comparative trial of P1496, a new non-steroidal oestrogen analogue. $\mathrm{Br}$ Med J 1973;i:579-81.

9 Fara GM, Del Corvo G, Bernuzzi S, et al. Epidemic of breast enlargement in an Italian school. Lancet 1979;ii:295-7.

10 Bongiovanni AM. An epidemic of premature thelarche in Puerto Rico. J Pediatr 1983;103:245-6.

11 Horwitz C, Rosen P, Gilat T. Should we worry about animal growth promoters? Nutr Cancer 1983;5:51-4.

12 Saenz de Rodriguez CA. Environmental hormone contamination in Puerto Rico. N Engl J Med 1984;310:1741-2.

13 Williams GM, Weisburger JH. Chemical carcinogens. In: Klassen C, Amdur MO, Doull J, eds. Casarett and Doull's toxicology: the basic science of poisons. New York: Macmillan, 1986:99-173.

14 Schoental $R$. Health hazards of secondary metabolites of Fusarium. Microbiologie-Aliments-Nutrition 1983;1:101-7.

15 National Toxicology Program. Carcinogenesis bioassay of zearalenone in F344/N rats and B6C3F, mice (feed study). Bethesda: National Institutes of Health, Public Health Service, US Department of Health and Human Services, 1982. (Tech report series No 235.)

16 Ruddick J, Scott P, Harwig J. Teratological evaluation of zearalenone administered orally to the rat. Bull Environ Contam Toxicol 1976;15:678-81.

17 Ueno Y, Kubota K. DNA-attacking ability of carcinogenic mycotoxins in recombinant-deficient mutant cells of Bacillus subtilis. Cancer Res 1986;36:445-51.

18 Kuczuk M, Benson P, Heath H, Hayes A. Evaluation of the mutagenic potential of mycotoxins using Salmonella typhimurium and Saccharomyces cerevisiae. Mutat Res 1978;53: $11-20$. 
19 Wehner F, Marasas W, Thiel P. Lack of mutagenicity to Salmonella typhimurium of some Fusarium mycotoxins. Appl Environ Microbiol 1978;35:659-62.

20 Aw TC, Stephenson RL, Smith AB. Cincinnati: NIOSH, 1984. (National Institute for Occupational Safety and Health, Health hazard evaluation report no HETA 82-257-1571.)

21 Harrington JM, Stein GF, Rivera RO, de Morales AV. The occupational hazards of formulating oral contraceptives-a survey of plant employees. Arch Environ Health 1978;33:12-5.

22 Harrington JM. Occupational exposure to synthetic estrogens: some methodological problems. Scand J Work Environ Health 1982;8(suppl 1):167-71.

23 Nuttall FQ. Gynecomastia as a physical finding in normal men. $J$ Clin Endocrinol Metab 1979;4:338-40.

24 Lipid Research Clinics Program. Manual of laboratory operations. Vol 1. Lipid and lipoprotein analysis. Washington: Government Printing Office, 1974. (DHEW publication No (NIH) 75-628.)

25 Schaefer EJ, Levy RI. Pathogenesis and management of lipoprotein disorders. $N$ Engl J Med 1985;312:1300-10.

26 Goisis C, Goisis F, Tammiso R. Estrogenic absorption through the skin: an experimental study. Acta Eur Fertil 1980;11:61-85.

27 Jeffcoate SJ. The hormonal peptides of the hypothalamus. In: Butt WR, ed. Topics in hormone chemistry. Vol 1. Chichester: Ellis
Horwood Ltd, 1978:13-47.

28 Daughaday WH. The anterior pituitary. In: Wilson JW, Fosto DW, eds. William's textbook of endocrinology. Philadelphia: WB Saunders, 1985:568-613.

29 Hochstrasser JM. Exposure to synthetic estrogens. Am Ind Hyg Assoc J 1978;39:675-7.

30 Imparatio E, Marino L, Sullusto A. Controlled clinical evaluatio of a new drug in the menopausal syndrome. Archivio Ostretucia e Ginecologia 1972;77:203-33.

31 Robynj C, Vekemans M, Delvoye P, L'Hermite M. Gonadotro@ phin and prolactin secretion during hormonal contraception In: Haspels AA, Kay CR, eds. International symposium on hormonal contraception. Amsterdam: Excerpta Medica, $1978 \dot{5}$ 98-125.

32 Lindsay DG. Zeranol-a "natural-identical" oestrogen? Food an Chemical Toxicology 1985;23:767-74.

33 Hoover R, Gray LA Sr, Cole P, Macmahon B. Menopausa estrogens and breast cancer. $N$ Engl J Med 1976;295:401-5. (D)

34 Zeil HK, Finkle WD. Increased risk of endometrial carcinoma among users of conjugated estrogens. $N$ Engl J Med 1975 293:1167-70.

35 Estrogen treatment of the young, supplement. Pediatrics 1978;62 1087-217.

\section{Correspondence and editorials}

The British Journal of Industrial Medicine welcomes correspondence relating to any of the material appearing in the journal. Results from preliminary or small scale studies may also be published in the correspondence column if this seems appropriate. Letters should be not more than 500 words in length and contain a minimum of references. Tables and figures should be kept to an absolute minimum. Letters are accepted on the understanding that they may be subject to editorial revision and shortening.

The journal now also publishes editorials which are normally specially commissioned. The Editor welcomes suggestions regarding suitable topics; those wishing to submit an editorial, however, should do so only after discussion with the Editor. 